\title{
Diagnóstico de pubertad precoz: Guía de práctica clínica para el diagnóstico y el tratamiento de la pubertad precoz
}

\author{
América L. Miranda-Lora ${ }^{1}$, Margarita Torres-Tamayo², Jessie N. Zurita-Cruz ${ }^{3}$, Blanca E. Aguilar-Herrera ${ }^{4}$, \\ Raúl Calzada-León ${ }^{5}$, Aleida J. Rivera-Hernández ${ }^{3}$, Marco A. Morales-Pérez ${ }^{6}$, Miriam M. Padrón-Martínez ${ }^{5}$, \\ María L. Ruiz-Reyes ${ }^{5}$, Leticia M. García-Morales ${ }^{1}$, Consuelo Barrón-Uribe ${ }^{4}$, Sletza L. Arguinzoniz-Valenzuela ${ }^{5}$, \\ Mayra C. Torres-Castañeda7, Lorena Lizárraga-Paulin7, Jorge A. Núñez-Hernández ${ }^{8,9}$, Judith Cornejo- \\ Barrera $^{10}$, María T. Vidal-González ${ }^{11}$, María R. Martínez-Alvarado², Elisa Nishimura-Meguro ${ }^{4}$, \\ Luz E. Bravo-Ríos ${ }^{4}$, Eulalia P. Garrido-Magaña ${ }^{3}$, José A. Orozco-Morales ${ }^{1}$, Patricia G. Medina-Bravo', \\ Ninel Coyote-Estrada y M. Fernanda Castilla-Peón ${ }^{1 *}$ \\ ${ }^{1}$ Hospital Infantil de México Federico Gómez, Ciudad de México; ${ }^{2}$ Instituto Nacional de Cardiología Ignacio Chávez, Ciudad de México; ${ }^{3} \mathrm{Hospital}$ \\ de Pediatría, Centro Médico Nacional Siglo XXI, Instituto Mexicano del Seguro Social (IMSS), Ciudad de México; ${ }^{4}$ Sociedad Mexicana de \\ Endocrinología Pediátrica; ${ }^{5}$ Instituto Nacional de Pediatría, Ciudad de México; ${ }^{6}$ Hospital General de Zona No. 18, IMSS, Playa del Carmen, Quintana \\ Roo; ${ }^{7}$ Hospital General, Centro Médico Nacional La Raza, IMSS, Ciudad de México; ${ }^{8}$ Hospital General Regional 220, IMSS, Estado de México; \\ 9 Instituto Materno Infantil del Estado de México, Toluca, Estado de México; ${ }^{10} \mathrm{Hospital}$ Infantil de Tamaulipas, Ciudad Victoria, Tamaulipas; ${ }^{11} \mathrm{Hospital}$ \\ del Niño y el Adolescente Morelense, Cuernavaca, Morelos. México
}

\section{Resumen}

La Sociedad Mexicana de Endocrinología Pediátrica elaboró una guía de práctica clínica para el diagnóstico y el tratamiento de la pubertad precoz. Este documento presenta recomendaciones relacionadas con el diagnóstico de pubertad precoz. La descripción detallada de la metodología para el desarrollo de esta guía y del sistema de gradación, así como la síntesis de la evidencia en la que se basa, pueden consultarse en este suplemento.

Palabras clave: Pubertad precoz. Diagnóstico. Hormona luteinizante. Hormonas esteroideas gonadales. Ultrasonido. Guía de práctica clínica.

\section{Diagnosis of precocious puberty: clinical guideline for the diagnosis and treatment of precocious puberty}

\begin{abstract}
The Mexican Society of Pediatric Endocrinology developed a clinical practice guide for the diagnosis and treatment of precocious puberty. This document presents recommendations related to the diagnosis of precocious puberty. The detailed description of the methodology for the development of this guide and the grading system, as well as the synthesis of the evidence on which it is based can be accessed in this same supplement.
\end{abstract}

Key words: Precocious puberty. Diagnosis. Luteinizing hormone. Gonadal steroid hormones. Ultrasonography. Clinical practice guideline.

Correspondencia:

*M. Fernanda Castilla-Peón

E-mail: fernandacastillapeon@gmail.com
Disponible en internet: 23-06-2020 Bol Med Hosp Infant Mex. 2020;77(Supl 1):7-14

www.bmhim.com 1665-1146/@ 2020 Hospital Infantil de México Federico Gómez. Publicado por Permanyer. Este es un artículo open access bajo la licencia CC BY-NC-ND (http://creativecommons.org/licenses/by-nc-nd/4.0/). 
La calidad de la evidencia de las recomendaciones acerca del diagnóstico se expresa con números romanos, de acuerdo con el Oxford Centre for EvidenceBased Medicine (OCEBM) ${ }^{1}$.

Recomendación 1.1. Se recomienda considerar la realización de estudios complementarios para el diagnóstico de pubertad precoz central en pacientes que inician con desarrollo de caracteres sexuales secundarios antes de los 8 años en niñas (telarca) y antes de los 9 años en niños (volumen testicular $\geq 4 \mathrm{~cm}^{3}$ ).

(Recomendación condicional I Nivel de evidencia: II OCEBM.)

\section{Evidencia}

El inicio de caracteres sexuales secundarios de forma prematura puede o no progresar a pubertad precoz central (PPC). Se ha descrito que del 21 al $64 \%$ de las niñas que inician con el desarrollo del botón mamario antes de los 8 años de edad presentan una telarca aislada y no evolucionan a $\mathrm{PPC}^{2,3}$. Junqueira, et al. ${ }^{3}$ reportaron una probabilidad del $36 \%$ de progresión de telarca aislada hacia PPC, del 50\% de pubarca prematura y del $72.2 \%$ de la combinación de ambas características. Otros hallazgos clínicos asociados a la PPC pueden ser la aceleración de la velocidad de crecimiento, la progresión de más de un estadio de Tanner en 6 meses o la no regresión de caracteres sexuales secundarios ${ }^{4}$. Con relación a esto, se ha descrito que la no regresión del tejido mamario presenta una sensibilidad (Sen) del $54.8 \%$, una especificidad (Esp) del $75.8 \%$, un valor predictivo positivo (VPP) del $37 \%$ y un valor predictivo negativo (VPN) del 87\% para identificar la progresión de la pubertad $^{5}$.

Considerando lo anterior, los criterios clínicos pueden no ser suficientes para establecer la presencia de PPC, por lo que se requieren estudios complementarios que ayuden a identificar las formas progresivas de pubertad en niñas con inicio de telarca antes de los 8 años y en niños con incremento del volumen testicular $\left(\geq 4 \mathrm{~cm}^{3}\right)$ antes de los 9 años.

Recomendación 1.2. Se recomienda evaluar la maduración esquelética como parte del abordaje inicial de los pacientes con sospecha de pubertad precoz central.

(Recomendación fuerte | Nivel de evidencia: || OCEBM.)

\section{Evidencia}

La evolución natural de la PPC involucra una aceleración inicial del crecimiento, pero con una disminución en la predicción de la estatura adulta que puede llegar a ser menor que la talla familiar esperada ${ }^{6}$. Lo anterior se relaciona con la maduración esquelética, por lo que uno de los hallazgos comunes en la pubertad precoz (PP) es el adelanto en la edad ósea (EO) con respecto a la edad cronológica (EC). Xu, et al. ${ }^{7}$ propusieron un punto de corte de 19.5 meses de diferencia entre la EO y la EC como predictor de PP (Sen $70 \%$, Esp $67 \%$ y falsos positivos $53 \%$ ). Calcaterra, et al. ${ }^{8}$ identificaron que una EO > 2 desviaciones estándar muestra una Sen del $72 \%$, una Esp del $74.3 \%$, un VPP del $67 \%$ y un VPN del $79 \%$ para identificar una $\mathrm{PPC}$ rápidamente progresiva en niñas. De Vries, et al. ${ }^{2}$ identificaron que un puntaje $Z$ de EO $>1$ presenta una Sen del $73 \%$, una Esp del $81 \%$, un VPP del $93.4 \%$ y un VPN del $47.3 \%$ para diferenciar entre PPC y telarca prematura.

De acuerdo con lo anterior, a pesar de que el adelanto en la EO no es un criterio suficiente para identificar la PPC, es un indicador que facilita el diagnóstico y debe formar parte del abordaje inicial de estos pacientes, lo que además repercutirá en el seguimiento y la evaluación de la respuesta al tratamiento.

Recomendación 1.3. Se sugiere documentar la
activación del eje hipotálamo-hipófisis-gónada para
confirmar el diagnóstico de pubertad precoz central
mediante la determinación de la hormona luteinizante
(LH) en sangre. Un valor de $\mathrm{LH} \geq 0.3$ UI/I determinado
por inmunoquimioluminiscencia puede ser conside-
rado como punto de corte adecuado para el diagnós-
tico de pubertad precoz central.
(Recomendación condicional I Nivel de evidencia: II
OCEBM.)

\section{Evidencia}

Debido a que la aparición de caracteres sexuales secundarios de forma prematura puede no progresar hacia PPC, se requiere documentar la activación del eje hipotálamo-hipófisis-gónada para la confirmación diagnóstica. Los estudios señalan que el incremento en los valores basales de hormona luteinizante (LH) aumenta la probabilidad de progresión hacia PPC. Derivado de lo anterior, se ha establecido que la determinación de la LH en una muestra aleatoria mediante ensayos ultrasensibles permite confirmar el diagnóstico 
Tabla 1. Estudios que evalúan el rendimiento diagnóstico de los valores basales de hormona luteinizante

\begin{tabular}{|c|c|c|c|c|c|c|}
\hline Punto de corte (UI/I) & Método analítico & $\operatorname{Sen}(\%)$ & Esp (\%) & VPP (\%) & VPN (\%) & Referencia \\
\hline 0.1 & \multirow[t]{2}{*}{ ICMA } & 92 & 88 & 90 & 90 & \multirow[t]{2}{*}{ Neely, et al., $1995^{10}$} \\
\hline$>0.3$ & & 79 & 100 & 100 & 80 & \\
\hline 0.6 (niños) & IFMA & 71 & 100 & 100 & 75 & \multirow[t]{2}{*}{ Brito, et al., 199913 } \\
\hline 0.6 (niñas) & ICMA & 63 & 100 & 100 & 41 & \\
\hline$>0.83$ & ICMA & 93 & 100 & ND & ND & Houk, et al., $2009^{11}$ \\
\hline$>0.1$ & ICMA & 76 & 80 & 82 & 74 & Poomthavorn, et al., $2009^{14}$ \\
\hline$>0.3$ & ICMA & 35 & 100 & 100 & 52 & Sathasivam, et al., $2010^{15}$ \\
\hline$>1.1$ & ICMA & 69 & 51 & 34 & 82 & Lee, et al., $2012^{16}$ \\
\hline$>0.1$ & ICMA & 64 & 96 & 93 & 71 & Pasternak, et al., $2012^{17}$ \\
\hline$>0.2$ & ICMA & 71 & 77 & 76 & 73 & De Filippo, et al., $2013^{18}$ \\
\hline$>0.2$ & ICMA & 89 & 100 & 100 & 95 & Harrington, et al., $2014^{12}$ \\
\hline$>0.1$ & ICMA & 71 & 94 & 91 & 80 & \multirow[t]{2}{*}{ Carreto, et al., $2014^{9}$} \\
\hline$>0.8$ & ICMA & 46 & 100 & 100 & 69 & \\
\hline$>0.132$ & ICMA & 65 & 53 & ND & ND & Chin, et al., $2015^{19}$ \\
\hline$>0.37$ & ICMA & 38 & 82 & 81 & 40 & Chen, et al., $2017^{20}$ \\
\hline
\end{tabular}

Esp: especificidad; ICMA: inmunoquimioluminiscencia; IFMA: inmunofluorescencia; ND: no definido; Sen: sensibilidad; VPN: valor predictivo negativo; VPP: valor predictivo positivo.

de PPC y es considerado el mejor parámetro bioquímico para este propósito ${ }^{9-11}$.

Diversos estudios han evaluado el rendimiento diagnóstico de los valores basales de $\mathrm{LH}$, planteándose distintos puntos de corte (Tabla 1) ${ }^{9-20}$. Algunos autores han propuesto valores bajos de LH para disminuir los falsos negativos ( $\mathrm{LH}>0.2 \mathrm{UI} / \mathrm{L}$, Sen $91 \%$ y Esp $100 \%)^{12}$, mientras que otros han señalado que puede existir una superposición entre estadios prepuberales y puberales, con valores entre 0.3 y $0.83 \mathrm{UI} / \mathrm{I}^{11}$. Sin embargo, un valor de $\mathrm{LH}>0.3 \mathrm{UI} / / \mathrm{l}$ puede ser considerado un punto de corte adecuado para establecer el diagnóstico de PP en conjunto con la presencia de datos clínicos. Se debe tener precaución en utilizar este criterio diagnóstico en niños menores de 2 años debido a que las concentraciones de LH son mayores durante la etapa de minipubertad, y pueden dar un falso diagnóstico de PPC ${ }^{21,22}$.

Se ha propuesto que la medición de la LH en la primera orina de la mañana puede mostrar una Sen del $75-83 \%$ y una Esp del $72-92.3 \%$ para identificar formas progresivas de $\mathrm{PP}^{23,24}$. Si bien podría considerarse una prueba menos invasiva que las determinaciones en sangre, la información sobre su utilidad es escasa y no existe experiencia suficiente en la estandarización de la técnica. Debido a lo anterior, la documentación de la activación del eje hipotálamo-hipófisis-gónada mediante la medición de la $\mathrm{LH}$ debe realizarse en muestras de sangre.

Recomendación 1.4. Se recomienda considerar las determinaciones basales de estradiol o testosterona en rangos puberales, así como la relación LH/FSH $>0.6$, como pruebas complementarias para el diagnóstico de pubertad precoz central, aunque no imprescindibles.

(Recomendación condicional I Nivel de evidencia: III OCEBM.)

\section{Evidencia}

Se ha reportado que los valores basales de hormona estimulante del folículo (FSH) > $2.4 \mathrm{UI} / \mathrm{I}$ muestran una Sen del $68.3-76 \%$ y una Esp del $86.4-94.7 \%$ para identificar la PPC ${ }^{17,25}$. En lo que respecta a la determinación basal de estradiol, se ha identificado que los valores que superan $10-21 \mathrm{pg} / \mathrm{ml}$ poseen una Sen del $39.1-66 \%$ y una Esp del $64.8-100 \%$ para la detección de PP en 
las niñas ${ }^{9,13,15,25}$, mientras que los valores de testosterona $\geq 19 \mathrm{ng} / \mathrm{dl}$ muestran una Sen del $73.1 \%$ en los niños $^{13}$. En lo que respecta a la relación LH/FSH, se ha descrito que valores $>0.05-0.62$ tienen una Sen del $47-91 \%$ y una Esp del $52-97 \%$ para identificar la PPC $^{9,17,20,25-27}$.

De acuerdo con estos estudios, los valores basales de FSH y de estradiol/testosterona, así como la relación LH/FSH, pueden permitir la detección de PPC. Sin embargo, su rendimiento es menor que el de la determinación basal de LH.

Recomendación 1.5. Se recomienda realizar la prueba de estimulación con un análogo de la $\mathrm{GnRH}$ en los casos de sospecha de pubertad precoz central cuando las determinaciones basales de LH sean $<0.3 \mathrm{UI} / \mathrm{l}$ y se considere la opción de supresión farmacológica.

(Recomendación condicional I Nivel de evidencia: ॥ OCEBM.)

\section{Evidencia}

Los valores bajos de LH, así como la determinación basal de otros parámetros bioquímicos (FSH, estradiol/testosterona), no muestran una Sen óptima que permita excluir el diagnóstico de PPC (recomendaciones 2.3 y 2.4$)^{9-20,25-27}$. Si bien no se recomienda realizar sistemáticamente la prueba de estimulación con un análogo de la hormona liberadora de gonadotropina a $(\mathrm{GnRH})$ en el abordaje inicial, se debe considerar su determinación en aquellos casos de sospecha de PPC cuyas determinaciones basales de $\mathrm{LH}$ no sean diagnósticas $(<0.3 \mathrm{UI} / \mathrm{l})$ y se requiera confirmar la presencia de PPC, principalmente en pacientes en quienes se considere la opción de supresión con aGnRH.

Se han descrito distintas pruebas de estímulo con aGnRH con el propósito de documentar la activación del eje hipotálamo-hipófisis-gónada, las cuales varían en el tipo de aGnRH utilizado (leuprorelina o triptorelina) y en la dosis. Las pruebas consisten en la determinación de LH, FSH y estradiol/testosterona tras un estímulo con un aGnRH acuoso. La determinación de la LH se considera el principal parámetro bioquímico para la evaluación de la prueba, aunque no existe un consenso con relación al tiempo de medición (de 1 a 3 $\mathrm{h}$ después del estímulo). Adicionalmente, existen variaciones en el tipo de ensayo utilizado para la determinación hormonal, como inmunoquimioluminiscencia o electroquimioluminiscencia. Una de las pruebas más descritas es el estímulo con un aGnRH acuoso (100 $\mu \mathrm{g})$ y determinación de la LH entre 30 y 60 minutos después del estímulo $0^{2,13,14,28-30}$.

Recomendación 1.6. Se recomienda considerar unos valores de $\mathrm{LH}>5 \mathrm{UI} / \mathrm{l}$ en las 1-3 horas posteriores al estímulo con un análogo acuoso de la $\mathrm{GnRH}$ como criterio diagnóstico para identificar pacientes con pubertad precoz central.

(Recomendación condicional I Nivel de evidencia: II OCEBM.)

\section{Evidencia}

No existe un consenso en la literatura sobre el punto de corte óptimo de la LH en la prueba de estimulación con un aGnRH. Lo anterior se debe a las diferencias en el tipo de estímulo, los tiempos de la toma de muestras y el ensayo utilizado. Sin embargo, un punto de corte $>5 \mathrm{UI} / \mathrm{I}$ tras 1-3 horas del estímulo con un aGnRH puede ser considerado adecuado para establecer el diagnóstico de PPC, debido su rendimiento diagnóstico y la consistencia entre los estudios (Tabla 2) 2,3,8,9,12-17,19,28-32.

Se ha propuesto el uso de análogos de depósito para evaluar la activación del eje hipotálamo-hipófisis-gonadal, pero la evidencia es débil. En 2004, Brito, et al. ${ }^{33}$ determinaron la $\mathrm{LH}$ a las 2 horas de la primera aplicación de $3.75 \mathrm{mg}$ de acetato de leuprorelina en 12 niñas, en quienes previamente se corroboró el diagnóstico de PPC mediante la prueba de estimulación con aGnRH acuoso. El estudio reportó una Sen del 100\% para valores de LH $>10 \mathrm{UI} / \mathrm{L}$ a las 2 horas; sin embargo, la metodología del estudio no permitió evaluar otros parámetros del rendimiento diagnóstico. Se requiere mayor evidencia para poder hacer una recomendación del uso de análogos de depósito en la prueba de estimulación, así como evaluar las repercusiones en pacientes en quienes no se corrobore el diagnóstico.

Recomendación 1.7. Se recomienda considerar
la medición del estradiol en las niñas a las
24 horas de la prueba de estimulación con un
análogo de la GnRH. Un punto de corte de estra-
diol > $50 \mathrm{pg} / \mathrm{ml}$ puede ser considerado como un
criterio diagnóstico adecuado para pubertad pre-
coz central. No se cuenta con evidencia para la
determinación de testosterona tras el estímulo en
los varones.
(Recomendación condicional I Nivel de evidencia:
III OCEBM.)


Tabla 2. Estudios que evalúan el rendimiento diagnóstico de la prueba de estimulación con análogos de la hormona liberadora de gonadotropina

\begin{tabular}{|c|c|c|c|c|c|c|c|}
\hline Estímulo & $\begin{array}{l}\text { Tiempo de } \\
\text { medición de pico } \\
\text { de LH (min) }\end{array}$ & $\begin{array}{l}\text { Punto de } \\
\text { corte (UI/I) }\end{array}$ & $\begin{array}{l}\text { Sen } \\
(\%)\end{array}$ & $\begin{array}{l}\text { Esp } \\
(\%)\end{array}$ & $\begin{array}{l}\text { VPP } \\
(\%)\end{array}$ & $\begin{array}{l}\text { VPN } \\
(\%)\end{array}$ & Referencia \\
\hline $100 \mu \mathrm{g}$ GnRH & $30,45,60,90$ & 15 & $92-95$ & $81-94$ & $92-97$ & $50-86$ & Cavallo, et al., $1995^{28}$ \\
\hline \multirow[t]{2}{*}{$100 \mu \mathrm{g}$ GnRH } & \multirow[t]{2}{*}{ Pico 15, 30, 45, 50} & 9.6 (niños) & 100 & 100 & 100 & 100 & \multirow[t]{2}{*}{ Brito, et al., $1999^{13}$} \\
\hline & & 6.9 (niñas) & 92 & 100 & 100 & 77 & \\
\hline $100 \mu \mathrm{g} \mathrm{GnRH}$ & Pico 0, 30, 50 & $>5$ & 63 & 96 & 98 & 41 & de Vries, et al., $2006^{2}$ \\
\hline \multirow[t]{4}{*}{$60 \mu \mathrm{g} / \mathrm{m}^{2} \mathrm{GnRH}$} & 15 & \multirow[t]{4}{*}{$>9$} & 89 & 90 & 92 & 85 & \multirow[t]{4}{*}{ Choi, et al., $2007^{31}$} \\
\hline & 30 & & 100 & 79 & 87 & 100 & \\
\hline & 60 & & 92 & 79 & 86 & 88 & \\
\hline & 90 & & 81 & 95 & 95 & 78 & \\
\hline $\mathrm{GnRH} ?$ & ND & 7 & 88 & 100 & 100 & 91 & Calcaterra, et al., $2009^{8}$ \\
\hline Triptorelina $0.1 \mathrm{mg}$ & 60 & $>6$ & 89 & 91 & 79 & 85 & Poomthavorn, et al., 200914 \\
\hline Leuprorelina acuosa $20 \mu \mathrm{g} / \mathrm{kg}$ & Pico 60 y 120 & $>5$ & 78 & 100 & 100 & 76 & Sathasivam, et al., $2010^{15}$ \\
\hline $100 \mu \mathrm{g} \mathrm{GnRH}$ & 40 & $>5$ & 98 & 100 & 100 & 74 & Kandemir, et al., $2011^{29}$ \\
\hline $100 \mu \mathrm{g} \mathrm{GnRH}$ & 45 & $>5$ & 98 & 100 & 100 & 95 & Kim, et al., $2011^{30}$ \\
\hline $\mathrm{GnRH}$ & ND & $>4.9$ & 78 & 79 & 80 & 77 & Pasternak, et al., $2012^{17}$ \\
\hline GnRH Triptorelina 0.1 mg/m² & 3 horas & $\begin{array}{l}>7 \mathrm{IFM} \\
>8 \mathrm{EQM}\end{array}$ & 76 & 100 & 100 & 62 & Freire, et al., $2013^{32}$ \\
\hline GnRH $50 \mu \mathrm{g}<1 \mathrm{~m}^{2}$ y $100 \mu \mathrm{g}>1 \mathrm{~m}^{2}$ & 60 & $>5$ & 94 & 90 & 81 & 97 & Harrington, et al., $2014^{12}$ \\
\hline Leuprorelina $500 \mu \mathrm{g}$ & 180 & $>5.5$ & 93 & 100 & 100 & 94 & Carretto, et al., $2014^{9}$ \\
\hline \multirow[t]{2}{*}{ Análogo de GnRH } & \multirow[t]{2}{*}{180} & 4 & 73 & 83 & 81 & 75 & \multirow[t]{2}{*}{ Junqueira, et al., $2015^{3}$} \\
\hline & & 8.4 & ND & 100 & ND & 100 & \\
\hline Estimulación $100 \mu \mathrm{g}$ GnRH & 180 & 5.1 & 75 & 87 & 88 & 72 & Chin, et al., $2015^{19}$ \\
\hline
\end{tabular}

EQM: electroquimioluminiscencia; Esp: especificidad; GnRH: hormona liberadora de gonadotropina; IFM: ensayo inmunofluorométrico; LH: hormona luteinizante; ND: no definido; Sen: sensibilidad; VPN: valor predictivo negativo; VPP: valor predictivo positivo.

\section{Evidencia}

La determinación del estradiol puede mejorar el rendimiento diagnóstico de la prueba de estímulo con un aGnRH cuando se realiza entre las 18 y 24 horas posteriores. Se ha reportado que los valores de estradiol $>52.9 \mathrm{pg} / \mathrm{ml}$ a las 24 horas del estímulo con un aGnRH muestran una Sen del $68-84 \%$ y una Esp del $74-100 \%$ para identificar PP ${ }^{3,15}$. Sathasivam, et al. ${ }^{15}$ reportaron que agregar la determinación del estradiol con un punto de corte $>50 \mathrm{pg} / \mathrm{ml}$ a la prueba de estimulación con un aGnRH permite alcanzar una Sen y una Esp del $100 \%$. Otros autores han reportado una Sen del $75-94 \%$ y una Esp del $97-100 \%$ al agregar los valores de estradiol $>80 \mathrm{pg} / \mathrm{ml}$ como criterio diagnóstico a la prueba de estimulación ${ }^{9,34}$. Chin, et al. ${ }^{19}$ reportaron que un incremento del $27.8 \%$ en los valores del estradiol en 3-24 horas tras la prueba de estimulación muestra una Sen del $80 \%$ y una Esp del $87 \%$ para identificar PP.

De acuerdo con lo anterior, la determinación del estradiol incrementa el rendimiento diagnóstico de la curva de estimulación con aGnRH, por lo que se puede considerar su determinación en los casos en que las características clínicas no concuerden con los estudios diagnósticos previos. Un valor de estradiol $>50 \mathrm{pg} / \mathrm{ml}$ a las 24 horas del estímulo con un aGnRH puede ser considerado un punto de corte adecuado para establecer el diagnóstico de PPC. 
Tabla 3. Parámetros ultrasonográficos y su rendimiento diagnóstico en pacientes con pubertad precoz central

\begin{tabular}{|c|c|c|c|}
\hline Parámetro ultrasonográfico & $\operatorname{Sen}(\%)$ & Esp (\%) & Referencias \\
\hline \multicolumn{4}{|l|}{ Útero } \\
\hline Longitud $>3-3.5 \mathrm{~cm}$ & $82-100$ & $86-100$ & $\begin{array}{l}\text { de Vries, et al., 2006²; Binay, et al., 2014 }{ }^{25} \text {; Supornsilchai, et al., } 2003^{26} \text {; } \\
\text { Badouraki, et al., 2008 }\end{array}$ \\
\hline Diámetro transverso $>1.5 \mathrm{~cm}$ & 67.9 & 100 & de Vries, et al., $2006^{2}$ \\
\hline Tamaño del fondo $>0.8 \mathrm{~cm}$ & 82.5 & 76.4 & de Vries, et al., $2006^{2}$ \\
\hline $\begin{array}{l}\text { Volumen uterino (puntos de } \\
\text { corte entre } 1.8 \text { y } 5 \mathrm{~cm}^{3} \text { ) }\end{array}$ & $52-100$ & $64-100$ & $\begin{array}{l}\text { de Vries, et al., } 2006^{2} \text {; Calcaterra, et al., 20098; Badouraki, et al., } 2008^{35} \text {; } \\
\text { Haber, et al., } 1995^{36} \text {; Battaglia, et al., } 2003^{38}\end{array}$ \\
\hline $\begin{array}{l}\text { Relación fondo/cérvix (puntos } \\
\text { de corte entre } 0.98 \text { y } 1.05 \text { ) }\end{array}$ & $29-91$ & $67-87$ & Binay, et al., 201425; Badouraki, et al., 200835; Eksioglu, et al., $2013^{39}$ \\
\hline Presencia de eco endometrial & $55-87.5$ & 74.2-86 & $\begin{array}{l}\text { de Vries, } 2006^{2} \text {; Calcaterra, et al., } 2009^{8} \text {; Battaglia, et al., } 2003^{38} \text {; } \\
\text { Eksioglu, et al., } 2013^{39}\end{array}$ \\
\hline $\begin{array}{l}\text { Índice de pulsatilidad de } \\
\text { arterias uterinas }>2.5\end{array}$ & $86-94$ & $96-100$ & Battaglia, et al., $2002^{37}$ \\
\hline \multicolumn{4}{|l|}{ Ovarios } \\
\hline Volumen $>1.2$ a $1.6 \mathrm{~cm}^{3}$ & $72-88$ & $63-95$ & Binay, et al., 201425; Badouraki, et al., 200835; Haber, et al., $1995^{36}$ \\
\hline Circunferencia $>4.5 \mathrm{~cm}$ & 67 & 86 & de Vries, et al., $2006^{2}$ \\
\hline
\end{tabular}

Esp: especificidad; Sen: sensibilidad.

Recomendación 1.8. Se recomienda considerar las características ultrasonográficas del útero y de los ovarios como apoyo diagnóstico de pubertad precoz central en aquellos casos con sospecha clínica en los que no se tenga la disponibilidad de realizar pruebas de estimulación con análogos de las $\mathrm{GnRH}$ y sin las concentraciones hormonales basales diagnósticas. (Recomendación condicional I Nivel de evidencia: III OCEBM.)

\section{Evidencia}

El ultrasonido pélvico no se considera un criterio diagnóstico para PPC. Sin embargo, el aumento del volumen ovárico $\left(>2 \mathrm{~cm}^{3}\right)$ refleja la estimulación de gonadotropinas y el crecimiento uterino ( $>3-5 \mathrm{~cm}$ de longitud) indica la estimulación estrogénica. Se han propuesto distintos parámetros ultrasonográficos para evaluar las características puberales de los genitales internos $2,7,8,25,35-39$. Como se muestra en la Tabla 3, existe una heterogeneidad importante en los parámetros ultrasonográficos utilizados y en los puntos de corte propuestos, aunado a que los resultados suelen ser dependientes del operador. Por lo anterior, el ultrasonido pélvico debe considerarse un estudio complementario y no una prueba suficiente para establecer el diagnóstico de PPC.
Recomendación 1.9. No se recomienda realizar ultrasonido mamario como prueba diagnóstica para pubertad precoz central.

(Recomendación condicionall Nivel de evidencia: III OCEBM.)

\section{Evidencia}

Se ha reportado que un volumen mamario $>0.85 \mathrm{~cm}^{3}$ posee una Sen del $66 \%$ y una Esp del $62 \%$ para la detección de PPC ${ }^{8}$. Debido a que la evidencia sobre su uso es escasa y a que no tiene ventajas sobre los datos clínicos y otras pruebas diagnósticas, no se recomienda su realización sistemática en pacientes con sospecha de PPC.

Recomendación 1.10. No se recomienda la determinación sistemática de androstenediona, hormona antimulleriana e inhibina B para establecer el diagnóstico de pubertad precoz central.

(Recomendación condicionall Nivel de evidencia: III OCEBM.)

\section{Evidencia}

Se ha reportado que unos valores de androstenediona $>1 \mathrm{mmol} / \mathrm{l}$ poseen una Sen del $59 \%$ y una Esp del $76 \%$ 
para diferenciar entre telarca prematura y PP; sin embargo, la información acerca de su rendimiento diagnóstico es escasa $^{2}$. Se ha observado una situación similar para la inhibina B. De Filippo, et al..$^{18}$ reportaron que los valores de inhibina $B>20 \mathrm{pg} / \mathrm{ml}$ muestran una Sen del $60 \%$ y una Esp del $89 \%$ para identificar formas progresivas de PPC como prueba aislada, y un mayor rendimiento combinando los resultados con los valores basales de LH > 0.2, alcanzando una Sen del $98 \%$ y una Esp del $98.7 \%$. Chen, et al. ${ }^{20}$ reportaron que los valores de hormona antimulleriana $>2.69 \mathrm{pmol} / \mathrm{l}$, en conjunto con la inhibina $\mathrm{B}>$ $30.12 \mathrm{pg} / \mathrm{ml}$, muestran una Sen del $80 \%$ y una Esp del $89.3 \%$ para distinguir entre formas progresivas y no progresivas de PPC. Dado que la determinación aislada de inhibina B (sola o en combinación con la hormona antimulleriana) no supera el rendimiento diagnóstico que se obtiene con la determinación de la LH basal o estimulada, que estas pruebas no están disponibles en la mayoría de los centros que atienden a la población blanco de esta guía, y que la información sobre su utilidad diagnóstica es escasa, no se recomienda su determinación sistemática para establecer el diagnóstico de PPC hasta que se cuente con mayor evidencia científica.

Recomendación 1.11. No se recomienda la realización de citología vaginal para establecer el diagnóstico de pubertad precoz central.

(Recomendación condicional I Nivel de evidencia: III OCEBM.)

\section{Evidencia}

Existe poca evidencia sobre la utilidad de la citología vaginal para el diagnóstico de PPC. Haber, et al. ${ }^{36}$ reportaron una Sen del $60 \%$ y una Esp del $63.2 \%$ para la detección de PPC. No se recomienda su realización debido a que es un procedimiento invasivo y existen otras pruebas con mayor rendimiento diagnóstico.

Recomendación 1.12. Se recomienda el seguimiento trimestral de aquellos pacientes en quienes la sospecha clínica y las pruebas diagnósticas disponibles no permitan confirmar ni descartar la presencia de pubertad precoz central. Adicionalmente, se deben realizar estudios de investigación con una adecuada calidad metodológica que evalúen el mejor abordaje diagnóstico y el uso racional de las pruebas complementarias para la detección oportuna y adecuada de los pacientes con pubertad precoz central.

(Buena práctica clínica | Nivel de evidencia: IV OCEBM.)

\section{Evidencia}

La evidencia científica actual muestra que existe heterogeneidad en el abordaje diagnóstico, los estudios complementarios y los puntos de corte utilizados para la adecuada clasificación de los pacientes con PPC. En la actualidad, el estándar de referencia continúa siendo la evolución clínica. Por ello, resulta indispensable el seguimiento de aquellos pacientes en quienes exista la sospecha clínica de PPC, pero que no cuenten con pruebas complementarias para confirmar o descartar el diagnóstico, ya sea por la falta de recursos o porque los resultados de las pruebas no permiten confirmar o rechazar el diagnóstico.

Dado que una conducta de vigilancia puede retrasar el inicio de intervenciones dirigidas a modificar el curso clínico del padecimiento, es necesario contar con el apoyo de pruebas diagnósticas confiables, por lo que se requiere realizar investigaciones futuras con diseños metodológicos óptimos que permitan mejorar la calidad de la evidencia científica actual.

\section{Bibliografía}

1. Torres-Tamayo M, Zurita-Cruz JN, Aguilar-Herrera BE, Miranda-Lora AL, Calzada-León R, Rivera Hernández AJ, et al. Metodología para la elaboración de la Guía de práctica clínica para el diagnóstico y el tratamiento de la pubertad precoz. Bol Med Hosp Infant Mex. 2020; 77(Supl. 1):1-6

2. de Vries L, Horev G, Schwartz M, Phillip M. Ultrasonographic and clinical parameters for early differentiation between precocious puberty and premature thelarche. Eur J Endocrinol. 2006;154:891-8.

3. Junqueira FR, Lara LA, Martins WP, Ferriani RA, Rosa ESAC, de Sá MF, et al. Gonadotropin and estradiol levels after leuprolide stimulation tests in Brazilian girls with precocious puberty. J Pediatr Adolesc Gynecol. 2015;28:313-6.

4. Volta C, Bernasconi S, Cisternino M, Buzi F, Ferzetti A, Street ME, et al. Isolated premature thelarche and thelarche variant: clinical and auxological follow-up of 119 girls. J Endocrinol Invest. 1998;21:180-3.

5. Zhu SY, Du ML, Huang TT. An analysis of predictive factors for the conversion from premature thelarche into complete central precocious puberty. J Pediatr Endocrinol Metab. 2008;21:533-8.

6. Carel JC, Léger J. Clinical practice. Precocious puberty. N Engl J Med. 2008;358:2366-77.

7. Xu YQ, Li GM, Li Y. Advanced bone age as an indicator facilitates the diagnosis of precocious puberty. J Pediatr (Rio J). 2018;94:69-75.

8. Calcaterra V, Sampaolo P, Klersy C, Larizza D, Alfei A, Brizzi V, et al. Utility of breast ultrasonography in the diagnostic work-up of precocious puberty and proposal of a prognostic index for identifying girls with rapidly progressive central precocious puberty. Ultrasound Obstet Gynecol. 2009;33:85-91.

9. Carretto F, Salinas-Vert I, Granada-Yvern ML, Murillo-Valles M, Gomez-Gomez C, Puig-Domingo M, et al. The usefulness of the leuprolide stimulation test as a diagnostic method of idiopathic central precocious puberty in girls. Horm Metab Res. 2014;46:959-63.

10. Neely EK, Wilson DM, Lee PA, Stene M, Hintz RL. Spontaneous serum gonadotropin concentrations in the evaluation of precocious puberty. $J$ Pediatr. 1995;127:47-52.

11. Houk CP, Kunselman AR, Lee PA. Adequacy of a single unstimulated luteinizing hormone level to diagnose central precocious puberty in girls. Pediatrics. 2009;123:e1059-63.

12. Harrington J, Palmert MR, Hamilton J. Use of local data to enhance uptake of published recommendations: an example from the diagnostic evaluation of precocious puberty. Arch Dis Child. 2014;99:15-20.

13. Brito VN, Batista MC, Borges MF, Latronico AC, Kohek MB, Thirone AC, et al. Diagnostic value of fluorometric assays in the evaluation of precocious puberty. J Clin Endocrinol Metab. 1999;84:3539-44. 
14. Poomthavorn $P$, Khlairit $P$, Mahachoklertwattana $P$. Subcutaneous gonadotropin-releasing hormone agonist (triptorelin) test for diagnosing precocious puberty. Horm Res. 2009;72:114-9.

15. Sathasivam A, Garibaldi L, Shapiro S, Godbold J, Rapaport R. Leuprolide stimulation testing for the evaluation of early female sexual maturation. Clin Endocrinol (Oxf). 2010;73:375-81.

16. Lee HS, Park HK, Ko JH, Kim YJ, Hwang JS. Utility of basal luteinizing hormone levels for detecting central precocious puberty in girls. Horm Metab Res. 2012;44:851-4.

17. Pasternak Y, Friger M, Loewenthal N, Haim A, Hershkovitz E. The utility of basal serum LH in prediction of central precocious puberty in girls. Eur J Endocrinol. 2012;166:295-9.

18. De Filippo G, Rendina D, Nazzaro A, Lonardo F, Bouvattier C, StrazzuIlo $\mathrm{P}$. Baseline inhibin $\mathrm{B}$ levels for diagnosis of central precocious puberty in girls. Horm Res Paediatr. 2013;80:207-12.

19. Chin VL, Cai Z, Lam L, Shah B, Zhou P. Evaluation of puberty by verifying spontaneous and stimulated gonadotropin values in girls. J Pediatr Endocrinol Metab. 2015:28:387-92

20. Chen T, Wu H, Xie R, Wang F, Chen X, Sun H, et al. Serum anti-Mullerian hormone and inhibin $B$ as potential markers for progressive central precocious puberty in girls. J Pediatr Adolesc Gynecol. 2017;30:362-6.

21. Vestergaard ET, Schjorring ME, Kamperis K, Petersen KK, Rittig S, Juul $A$, et al. The follicle-stimulating hormone (FSH) and luteinizing hormone $(\mathrm{LH})$ response to a gonadotropin-releasing hormone analogue test in healthy prepubertal girls aged 10 months to 6 years. Eur $\mathrm{J}$ Endocrinol. 2017;176:747-53.

22. Bizzarri C, Spadoni GL, Bottaro G, Montanari G, Giannone G, Cappa M et al. The response to gonadotropin releasing hormone $(\mathrm{GnRH})$ stimulation test does not predict the progression to true precocious puberty in girls with onset of premature thelarche in the first three years of life. $J$ Clin Endocrinol Metabol. 2013;99:433-9.

23. Kolby $\mathrm{N}$, Busch AS, Aksglaede L, Sorensen K, Petersen JH, Andersson AM, et al. Nocturnal urinary excretion of FSH and LH in children and adolescents with normal and early puberty. J Clin Endocrino Metab. 2017:102:3830-8.

24. Zung A, Burundukov E, Ulman M, Glaser T, Rosenberg M, Chen M, et al. The diagnostic value of first-voided urinary LH compared with GNRH-stimulated gonadotropins in differentiating slowly progressive from rapidly progressive precocious puberty in girls. Eur J Endocrinol. 2014:170:749-58.

25. Binay C, Simsek E, Bal C. The correlation between GnRH stimulation testing and obstetric ultrasonographic parameters in precocious puberty J Pediatr Endocrinol Metab. 2014:27:1193-9.

26. Supornsilchai $V$, Hiranrat $P$, Wacharasindhu $S$, Srivuthana $S$, Aroonparkmon$\mathrm{gkol} \mathrm{S}$. Basal luteinizing hormone/follicle stimulating hormone ratio in diagnosis of central precocious puberty. J Med Assoc Thai. 2003;86:S145-51.
27. Wacharasindhu S, Srivuthana S, Aroonparkmongkol S, Shotelersuk V. A cost-benefit of $\mathrm{GnRH}$ stimulation test in diagnosis of central precocious puberty (CPP). J Med Assoc Thai. 2000;83:1105-11.

28. Cavallo A, Richards GE, Busey S, Michaels SE. A simplified gonadotrophin-releasing hormone test for precocious puberty. Clin Endocrinol (Oxf). 1995;42:641-6.

29. Kandemir N, Demirbilek H, Ozon ZA, Gonc N, Alikasifoglu A. GnRH stimulation test in precocious puberty: single sample is adequate for diagnosis and dose adjustment. J Clin Res Pediatr Endocrinol. 2011;3:12-7.

30. Kim HK, Kee SJ, Seo JY, Yang EM, Chae HJ, Kim CJ. Gonadotropin-releasing hormone stimulation test for precocious puberty. Korean J Lab Med. 2011;31:244-9.

31. Choi JH, Shin YL, Yoo HW. Predictive factors for organic central precocious puberty and utility of simplified gonadotropin-releasing hormone tests. Pediatr Int. 2007;49:806-10.

32. Freire AV, Escobar ME, Gryngarten MG, Arcari AJ, Ballerini MG, Bergadá I, et al. High diagnostic accuracy of subcutaneous triptorelin test compared with $\mathrm{GnRH}$ test for diagnosing central precocious puberty in girls. Clin Endocrinol (Oxf). 2013;78:398-404.

33. Brito VN, Latronico AC, Arnhold IJ, Mendonca BB. A single luteinizing hormone determination 2 hours after depot leuprolide is useful for therapy monitoring of gonadotropin-dependent precocious puberty in girls. $J$ Clin Endocrinol Metab. 2004:89:4338-42.

34. Freire AV, Gryngarten MG, Ballerini MG, Arcari AJ, Escobar ME, Bergada I, et al. Assessment of estradiol response after depot triptorelin administration in girls with central precocious puberty. Horm Res Paediatr. 2016;85:58-64.

35. Badouraki M, Christoforidis A, Economou I, Dimitriadis AS, Katzos G. Evaluation of pelvic ultrasonography in the diagnosis and differentiation of various forms of sexual precocity in girls. Ultrasound Obstet Gynecol. 2008;32:819-27.

36. Haber HP, Wollmann HA, Ranke MB. Pelvic ultrasonography: early differentiation between isolated premature thelarche and central precocious puberty. Eur J Pediatr. 1995;154:182-6.

37. Battaglia C, Regnani G, Mancini F, lughetti L, Venturoli S, Flamigni C. Pelvic sonography and uterine artery color Doppler analysis in the diagnosis of female precocious puberty. Ultrasound Obstet Gynecol. 2002;19:386-91.

38. Battaglia C, Mancini F, Regnani G, Persico N, lughetti L, De Aloysio D. Pelvic ultrasound and color Doppler findings in different isosexual precocities. Ultrasound Obstet Gynecol. 2003:22:277-83.

39. Eksioglu AS, Yilmaz S, Cetinkaya S, Cinar G, Yildiz YT, Aycan Z. Value of pelvic sonography in the diagnosis of various forms of precocious puberty in girls. J Clin Ultrasound. 2013;41:84-93. 